\title{
Cost-effectiveness of Drugs to Treat Relapsed/Refractory Multiple Myeloma in the United States
}

\author{
Josh J. Carlson, MPH, PhD; Gregory F. Guzauskas, MSPH, PhD; Richard H. Chapman, PhD, MS; \\ Patricia G. Synnott, MS, MALD; Shanshan Liu, MS; Elizabeth T. Russo, MD; \\ Steven D. Pearson, MD, MSc; Elizabeth D. Brouwer, MPH; and Daniel A. Ollendorf, PhD
}

\begin{abstract}
BACKGROUND: New 3-drug regimens have been developed and approved to treat multiple myeloma (MM). The absence of direct comparative data and the high cost of treatment support the need to assess the relative clinical and economic outcomes across all approved regimens.

OBJECTIVE: To evaluate the cost-effectiveness of treatments for relapsed and/or refractory MM from a U.S. health system perspective.

METHODS: We developed a partition survival model with 3 health states (progression-free, progression, and death) to evaluate the following regimens: carfilzomib (CFZ), elotuzumab (EL0), ixazomib (IX), daratumumab (DAR), and panobinostat (PAN) in combination with lenalidomide (LEN) or bortezomib (BOR) plus dexamethasone (DEX) in the second and/or third line of therapy. To estimate relative treatment effects, we developed a network meta-analysis and applied progression-free survival hazard ratios to baseline parametric progression-free survival functions derived from pooled data on LEN+DEX. We estimated overall survival using data on the relationship between progression-free survival and overall survival from a large meta-analysis of MM patients. Modeled costs included those related to drug treatment, administration, monitoring, adverse events, and progression. Utilities were from publicly available data and manufacturer data, if published sources were unavailable.
\end{abstract}

RESULTS: Model results showed that regimens containing DAR yielded the highest expected life years (DAR range: $6.71-7.38$ vs. non-DAR range: 3.25-5.27) and quality-adjusted life-years (QALY; DAR range: 4.38-5.44 vs. non-DAR range: 2.04-3.46), with DAR+BOR+DEX (second line) and $\mathrm{PAN}+\mathrm{BOR}+\mathrm{DEX}$ (third line) as the most cost-effective options (incremental cost-effectiveness ratio: $\$ 50,700$ and cost saving, respectively). The applicability of the PAN+BOR+DEX result may be challenging, however, because of ongoing toxicity concerns. In the probabilistic sensitivity analysis, second-line DAR+BOR+DEX and third-line PAN+BOR+DEX had an $89 \%$ and $87 \%$ probability of being cost-effective at the $\$ 150,000$ per QALY threshold, respectively.

CONCLUSIONS: The introduction of newer drugs and regimens to treat second- and third-line relapsed/refractory MM appears to provide clinical benefits by lengthening progression-free and overall survival and improving quality of life. However, only the addition of DAR or PAN may be considered cost-effective options according to commonly cited thresholds, and PAN+BOR+DEX results require cautious interpretation. Achieving levels of value more closely aligned with patient benefit would require substantial discounts from the remaining agents evaluated.

J Manag Care Spec Pharm. 2018;24(1):29-38

Copyright @ 2018, Academy of Managed Care Pharmacy. All rights reserved.

\section{What is already known about this subject}

Multiple myeloma (MM) treatment has historically been anchored by 2 drugs, bortezomib and lenalidomide, each in combination with dexamethasone.

Over the past decade, 6 more drugs entered the market and have demonstrated improved outcomes compared with standard care.

\section{What this study adds}

This study models comparative clinical and economic outcomes of combination drug therapies for MM treatment.

There is considerable uncertainty about comparative long-term outcomes, such as overall survival and the comparative trade-offs between effectiveness, toxicity, and costs for these therapies and their various combinations.

This study provides a comprehensive analysis of the value of these drug combinations and can inform health care decision making for a variety of stakeholders.

$\mathrm{M}$ ultiple myeloma (MM) is a hematological cancer that occurs when bone marrow produces malignant -plasma cells that enter the bloodstream. MM is the second most common hematological malignancy, with 25,000 new cases diagnosed every year in the United States. ${ }^{1}$ The disease disproportionately affects older people, with the median age of onset at 66 years. ${ }^{1}$ Despite recent advances, prognosis remains relatively poor, with a 5 -year survival rate of $48.5 \%{ }^{{ }^{1}}$ MM progression can be relatively slow in many individuals, often involving multiple rounds of remission after treatment followed by subsequent relapse. About 100,000 individuals are currently living with the disease in the United States. ${ }^{1}$

Over the past decade, treatment of MM in the United States has been anchored by 2 drugs: bortezomib (Velcade; BOR) and lenalidomide (Revlimid; LEN), often given in combination with dexamethasone (DEX). Other medications have more recently become available specifically for the treatment of relapsed or refractory disease, including pomalidomide (Pomalyst; POM), carfilzomib (Kyprolis; CFZ), ixazomib (Ninlaro; IX), daratumumab (Darzalex; DAR), elotuzumab (Empliciti; ELO), and panobinostat (Farydak; PAN)

These new agents have demonstrated improved outcomes compared with standard care approaches and are recommended as treatment options by national clinical guidelines. ${ }^{2}$ Collectively, they represent important clinical advances in a disease setting that has historically lacked a variety of treatment options. However, there remains considerable uncertainty regarding the comparative long-term outcomes (i.e., overall survival) and the comparative trade-offs between effectiveness and toxicity. In addition, there is considerable 
concern and uncertainty about the cost and value of these therapies, since the cost of a single course of drug therapy is estimated to range from $\$ 75,000$ to $\$ 250,000$ for U.S. patients with relapsed and/or refractory disease. ${ }^{3}$ These estimates may actually be conservative, given the increasing use of triple therapy and "treat to progression" labeling for the newest agents. ${ }^{3}$

The increasing cost of U.S. health care, and specifically for cancer, has generated renewed discussion about the value of medical technologies. The availability of effective treatment options for MM patients is of paramount importance. However, in an era of continuing increases in health care spending and drug prices, it is also important to understand the relationship between costs and outcomes achieved.

The objective of this study was to assess the comparative clinical and economic outcomes for drugs used to treat patients with relapsed and/or refractory MM in the second or third line of therapy from a U.S. health system perspective. This analysis can be used to inform health care decision making for a wide range of MM stakeholders.

\section{Methods}

\section{Model Approach}

The following pharmaceutical regimens were included in the analysis: LEN+DEX, BOR+DEX, CFZ+LEN+DEX, ELO+LEN+DEX, IX+LEN+DEX, PAN+BOR+DEX, DAR+LEN+DEX, and DAR+BOR+DEX (Table 1). We developed a 3-state partition survival model, which included a progression-free survival (PFS) state, progressed disease with subsequent treatments, and death. Patients in the PFS state could be either on or off treatment to account for patients who stop therapy but remain in the PFS state. We used a cycle length of 1 week to reflect the dosing schedules for included drug regimens. The recommended dosage schedules for the regimens of interest were based on indications for treatment of relapsed and/or refractory disease labeled by the U.S. Food and Drug Adminstration, as well as expert input regarding common treatment approaches for the populations of interest. We used a health sector perspective, a lifetime horizon, a 3\% discount rate for costs and outcomes, and a half-cycle correction. The upper bound willingness to pay for cost-effectiveness was $\$ 150,000$ per quality-adjusted life-years (QALY) gained, which approaches the upper limit of commonly cited thresholds. ${ }^{4}$

\section{Network Meta-analysis}

A Bayesian network meta-analysis (NMA) was performed to combine evidence on direct and indirect comparisons across the treatment regimens of interest (Appendix A, available in online article). ${ }^{5}$ The evaluated trials specified similar inclusion criteria. Each trial included adult patients aged $\geq 18$ years with measurable relapsed and/or refractory MM. All patients had previously received therapies and had adequate renal, hepatic, and hematologic function. The trial populations were also similar with respect to age, Eastern Cooperative Oncology Group
(ECOG) performance status, International Staging System (ISS) stage, receipt of previous stem cell transplant, and number and distribution of previous regimens.

Quantitative analyses focused on PFS and were conducted using the NetMetaXL tool (http://www.netmetaxl. $\mathrm{com} /$ ). Adjusted hazard ratios from the randomized trials were log-transformed and entered into the spreadsheet, and 95\% confidence intervals (CIs) on log scale were used to specify variance estimates (i.e., standard errors) ${ }^{6-15}$ A total of 40,000 iterations each were used for "burn-in" (for model convergence) and model (for model results) simulations. Review of the deviance information criterion statistics, as well as comparison of the residual deviance with the number of unconstrained data points, was used to assess the best model fit under multiple alternative assumptions. Although a random effects approach was preferred, the available network was constructed of primarily single-study connections and necessitated a fixed-effects model to preserve statistically significant effects observed in trials.

We also conducted sensitivity analyses based on the shape and scale parameters of digitized parametric survival curves to test and address the potential violation of the proportional hazards assumption. ${ }^{16}$ We did this for the overall dataset and a subset of data from the carfilzomib, ixazomib, and elotuzumab trials to assess whether inclusion of more contemporary data for LEN+DEX had a material effect on results. In this instance, 30,000 iterations were used for burn-in and model simulations.

\section{Survival Curve Estimation}

We fit parametric survival curves to PFS Kaplan-Meier data for the baseline comparator (LEN+DEX) in the second- and third-line settings, using the approach described by Hoyle and Henley (2011). ${ }^{17}$ LEN+DEX was chosen as the baseline because clinical experts considered LEN+DEX to be the most commonly used comparator and because of the availability of LEN+DEX survival data by line of therapy. To do this, we extracted data points from digitized survival curves, then used the extracted values, the number of surviving patients at each time interval, and maximum likelihood functions to estimate the underlying individual patient data. ${ }^{7,8,18}$ We assumed that the rate of censoring was the same between the second- and third-line settings, which allowed us to estimate the number at risk at set time points for the second-and third-line curves from the pooled number at risk data.

Base case PFS curves for LEN+DEX were derived from parametric fits to pooled Kaplan-Meier data from the MM-009 and MM-010 trials of LEN+DEX. ${ }^{7,8}$ For the base case, we selected the Weibull parametric function from the candidate distributions based on (a) face validity (log-normal and log-logistic fits exhibited unrealistically elongated tails) and (b) Akaike Information Criterion, a graphical assessment of each parametric function and a knowledge of the expected extrapolation of the PFS times. We then used PFS hazard ratios acquired 
TABLE 1 Key Model Parameters

\begin{tabular}{|c|c|c|c|c|c|}
\hline Survival Hazard Ratios & Base Case & Lower & Upper & PSA Distribution & Source \\
\hline \multicolumn{6}{|c|}{ Second-line PFS hazard ratios vs. LEN-DEX } \\
\hline CFZ-LEN-DEX & 0.69 & 0.53 & 0.91 & LogNormal & Network meta-analysis \\
\hline ELO-LEN-DEX & 0.70 & 0.56 & 1.00 & LogNormal & Network meta-analysis \\
\hline IX-LEN-DEX & 0.74 & 0.65 & 1.19 & LogNormal & Network meta-analysis \\
\hline DAR-LEN-DEXa & 0.37 & 0.27 & 0.52 & LogNormal & Network meta-analysis \\
\hline DAR-BOR-DEX ${ }^{a}$ & 0.39 & 0.28 & 0.53 & LogNormal & Network meta-analysis \\
\hline \multicolumn{6}{|c|}{ Third-line PFS hazard ratios vs. LEN-DEX } \\
\hline BOR-DEX & 0.93 & 0.58 & 2.04 & LogNormal & Network meta-analysis \\
\hline CFZ-LEN-DEX & 0.69 & 0.54 & 0.87 & LogNormal & Network meta-analysis \\
\hline ELO-LEN-DEX & 0.70 & 0.49 & 0.87 & LogNormal & Network meta-analysis \\
\hline IX-LEN-DEX & 0.74 & 0.40 & 0.84 & LogNormal & Network meta-analysis \\
\hline PAN-BOR-DEX & 0.59 & 0.31 & 1.10 & LogNormal & Network meta-analysis \\
\hline DAR-LEN-DEXa & 0.37 & 0.27 & 0.52 & LogNormal & Network meta-analysis \\
\hline DAR-BOR-DEXa & 0.39 & 0.28 & 0.53 & LogNormal & Network meta-analysis \\
\hline Hazard ratio for OS vs. PFS & 0.41 & 0.31 & 0.58 & LogNormal & 24 \\
\hline Quality of Life & Base Case & Lower & Upper & PSA Distribution & Source \\
\hline \multicolumn{6}{|l|}{ Second-line health state utilities } \\
\hline Progression-free, on treatment & 0.82 & 0.78 & 0.88 & Beta & Data on file ${ }^{b}$ \\
\hline Progression-free, off treatment & 0.84 & 0.82 & 0.97 & Beta & Data on file ${ }^{b}$ \\
\hline Progressed disease & 0.65 & 0.62 & 0.74 & Beta & Data on file ${ }^{b}$ \\
\hline \multicolumn{6}{|l|}{ Third-line health state utilities } \\
\hline Progression-free, on treatment & 0.65 & 0.52 & 0.78 & Beta & 25 \\
\hline Progression-free, off treatment & 0.72 & 0.58 & 0.86 & Beta & 37 \\
\hline Progressed disease & 0.61 & 0.49 & 0.73 & Beta & 25 \\
\hline Adverse event disutility & 0.08 & 0.07 & 0.08 & Beta & 25 \\
\hline Costs & Base Case & Lower & Upper & PSA Distribution & Source \\
\hline \multicolumn{6}{|c|}{ Drug acquisition and administration costs, ${ }^{\mathrm{c}} \$$} \\
\hline Bortezomib $3.5 \mathrm{mg}$ vial & $1,503.00$ & $1,202.40$ & $1,803.60$ & Normal & RED BOOK \\
\hline Bortezomib administration & 111.42 & 89.14 & 133.70 & Normal & CPT 96409 \\
\hline Carfilzomib $60 \mathrm{mg}$ vial & $1,971.50$ & $1,577.20$ & $2,365.80$ & Normal & RED BOOK \\
\hline Carfilzomib administration & 209.24 & 167.39 & 251.09 & Normal & CPT 96360, 96361, 96413 \\
\hline Dexamethasone per mg & 0.32 & 0.26 & 0.39 & Normal & RED BOOK \\
\hline Elotuzumab $300 \mathrm{mg}$ vial & $1,776.00$ & $1,420.80$ & $2,131.20$ & Normal & RED BOOK \\
\hline Elotuzumab $400 \mathrm{mg}$ vial & $2,368.00$ & $1,894.40$ & $2,841.60$ & Normal & RED BOOK \\
\hline Elotuzumab administration & 227.87 & 182.30 & 273.44 & Normal & CPT 96413, 96415, 96417 \\
\hline Ixazomib capsule & $3,006.00$ & $2,404.80$ & $3,607.20$ & Normal & RED BOOK \\
\hline Lenalidomide capsule & 552.98 & 442.38 & 663.58 & Normal & RED BOOK \\
\hline Panobinostat capsule & $1,222.22$ & 977.78 & $1,466.67$ & Normal & RED BOOK \\
\hline Daratumumab 400 mg vial & $1,850.40$ & $1,480.32$ & $2,220.48$ & Normal & RED BOOK \\
\hline Daratumumab 100 mg vial & 462.60 & 370.08 & 555.12 & Normal & RED BOOK \\
\hline Daratumumab administration & 399.83 & 319.86 & 479.80 & Normal & CPT 96413, 96415, 96417 \\
\hline Health State Cost per Week & \multicolumn{3}{|c|}{ Progression-Free AE Prophylaxis, \$ } & \multicolumn{2}{|c|}{ Progressed Disease Treatment, $\$$} \\
\hline LEN-DEX & \multicolumn{3}{|c|}{22.00} & \multicolumn{2}{|c|}{427.45} \\
\hline CFZ-LEN-DEX & \multicolumn{3}{|c|}{104.73} & \multicolumn{2}{|r|}{367.30} \\
\hline ELO-LEN-DEX & \multicolumn{3}{|c|}{85.37} & \multicolumn{2}{|r|}{369.26} \\
\hline IX-LEN-DEX & \multicolumn{3}{|c|}{85.26} & \multicolumn{2}{|r|}{377.05} \\
\hline PAN-BOR-DEX & \multicolumn{3}{|c|}{39.16} & \multicolumn{2}{|r|}{337.41} \\
\hline DAR-LEN-DEX & \multicolumn{3}{|c|}{49.45} & \multicolumn{2}{|r|}{273.54} \\
\hline DAR-BOR-DEX & \multicolumn{3}{|c|}{90.71} & \multicolumn{2}{|r|}{283.94} \\
\hline
\end{tabular}

aDAR hazard ratios were assessed for a general population and were assumed to be equivalent in second-and third-line settings.

bAmgen. Data provided in response to ICER data request. QOL/Utility Data from ASPIRE Cost Effectiveness Model. February 22, 2016.

${ }^{c}$ Costs assessed March 2016.

$B O R=$ bortezomib; $C F Z=$ carfilzomib; $C P T=$ Current Procedural Terminology; $D A R=$ daratumumab; $D E X=$ dexamethasone; ELO =elotuzumab; IX=ixazomib; LEN = lenalidomide; $O S=$ overall survival; $P A N=$ panobinostat $P F S=$ progression-free survival; $P S A=$ probabilistic sensitivity analysis. 
from our network meta-analysis results, applied to the baseline comparator curve (LEN+DEX), to derive PFS curves for the other interventions by line of treatment (Table 1).,13,14,19-23 We assumed that the regimens' NMA-derived treatment effects were consistent for the second- and third-line settings, since stratified analyses suggested similar effect sizes for most comparisons (data not shown).

The data on overall survival (OS) for these regimens were not uniformly available and were prone to bias because of crossover to the active comparator, as well as the availability of different drugs after progression in trials conducted at different points in time. Therefore, we applied a 2.45-month (95\% $\mathrm{CI}=1.7-3.2)$ increase in OS for each additional month of PFS for each regimen-specific OS curve, based on a systematic review of this relationship in studies of nearly 23,000 MM patients. ${ }^{24}$ This approach has been used previously in support of model submissions to health technology assessment agencies. ${ }^{25} \mathrm{We}$ operationalized this estimate by deriving an OS to PFS hazard ratio $(1 / 2.45=0.41)$, which we then applied to each regimen's PFS curve to estimate the corresponding OS curve. We varied this parameter in a sensitivity analysis and ran a scenario analysis using a weighted average estimate of the relationship of PFS to OS from the available clinical trials in our assessment (3.27month increase in OS for each additional month of median PFS).

\section{Model Parameters: Health State Utilities}

Health state utilities were derived from publicly available literature and/or data made available to us by manufacturers. We used consistent, sequence-specific health state utility values across treatments evaluated in the model. For the progressionfree health state, different utilities were applied, depending on whether the patient was on or off treatment, to represent treatment-related decreased quality of life. We applied a regimen-weighted disutility for experiencing any grade $3 / 4$ adverse event (AE). The total percentage of patients who experienced any grade 3/4 AEs for each regimen was multiplied by the AE disutility and then subtracted from the total QALYs gained during PFS for each regimen. We assumed that the total time that patients experienced any grade 3/4 AE was 1 month.

\section{Model Parameters: Adverse Events}

The model included grade 3/4 AEs derived from key clinical trials and/or each drug's prescribing information. The model included any reported grade 3/4 AEs that occurred in at least $5 \%$ of patients for any of the treatment comparators. ${ }^{3,26}$ The frequency of each event was reported as the proportion of patients experiencing the event, as well as the total number of occurrences based on published sources (Appendix B, available in online article). This proportion was then multiplied by the average cost per event to derive a total $\mathrm{AE}$ cost for each regimen. Costs per AE were based on a previously published analysis, supplemented by data from the Centers for Medicare $\&$ Medicaid Services (CMS) list of Medicare Severity-Diagnosis Related Groups (MS-DRGs) for the fiscal year 2016.3,26

\section{Model Parameters: Cost Inputs}

All patients were assumed to initiate study medications at cycle 0 (beginning of the model). For all regimens, patients received planned doses based on individual patient characteristics. The estimation of drug use was derived from several factors, including the relative dose intensity reported in trials or directly provided by manufacturers and the dosing schedule where the dose may be fixed by weight or by body surface area (BSA), assuming average patient characteristics of 1.7 meters tall and 80 kilogram body mass (BSA $=1.92$; formula: $0.007184 *[$ mass $\wedge 0.425]^{*}\left[\right.$ height $\left.{ }^{\wedge} 0.725\right]$; unpublished data. Amgen. Data provided in response to ICER data request. QOL/Utility Data from ASPIRE Cost Effectiveness Model. February 22, 2016).

The treatment utilization and costs of treat-to-progression regimens were applied to all patients who remained in the PFS health state over time. In finite-cycle regimens, patients could remain in the PFS state after active treatment concluded. No vial sharing was assumed in the base case. Drug unit costs were applied to the utilization estimates to calculate total estimated treatment costs.

We used the wholesale acquisition cost for each drug and noted each available formulation. ${ }^{25}$ Based on the regimenspecific dosage previously specified, the model used the lowest cost combination of tablets and/or vials for each regimen. Drug administration costs were determined from the Final 2016 Medicare Coding \& Payment for Drug Administration Services under the Physician Fee Schedule. ${ }^{27}$ All drug and administration costs were varied by $\pm 20 \%$ in sensitivity analyses.

Other Direct Costs. We included per cycle AE prophylaxis and monitoring costs based on management guidelines and the literature. ${ }^{6}$ The cost of postprogression treatment was calculated using a treatment landscape analysis to estimate the proportion of patients who received different available treatments upon progression. The specific treatment distribution was derived from Farr et al. (2016). ${ }^{28}$ The model assumed that patients received 1 further line of treatment lasting 124 days followed by best supportive care. ${ }^{28}$ We then calculated a mean cost per week for each regimen by averaging the cost of additional treatment (weighted by the distribution) and supportive care over the mean time in the progression health state.

\section{Model Outcomes}

The model estimated the amount of time, on average, that patients spent progression-free and in progression. Unadjusted and utility-adjusted time spent in each health state were summed to provide estimates of life expectancy and qualityadjusted life expectancy. Model outcomes of interest for each intervention included cost, quality-adjusted life expectancy, life expectancy, and mean time in the progression-free and postprogression health states, as well as the relative costeffectiveness for each intervention compared with the next best comparator. We also provided the results of pairwise comparisons by line versus a standard comparator, LEN-DEX. 
Cost-effectiveness of Drugs to Treat Relapsed/Refractory Multiple Myeloma in the United States

\section{TABLE 2 Comparative Outcomes}

\begin{tabular}{l|c|c|c|c|c|c|c|c|c}
\hline Regimen & \multicolumn{3}{|c|}{ Second Line } & \multicolumn{3}{c|}{ Third Line (All Comparators) } & \multicolumn{2}{c}{ Third Line (PAN-BOR-DEX Omitted) } \\
\hline & Total Cost, $\$$ & QALYs & ICER & Total Cost, $\$$ & QALYs & ICER & Total Cost, \$ & QALYs & ICER \\
\hline LEN-DEX & 309,997 & 2.59 & Dominated & 281,754 & 2.04 & Dominated & 281,754 & 2.04 & Dominated \\
\hline BOR-DEX & 189,357 & 2.74 & Dominant & 175,315 & 2.16 & Dominant & 175,315 & 2.16 & Dominant \\
\hline IX-LEN-DEX & 622,378 & 3.27 & Dominated & 566,512 & 2.60 & Dominated & 566,512 & 2.60 & Dominated \\
\hline ELO-LEN-DEX & 665,728 & 3.41 & Dominated & 608,651 & 2.71 & Dominated & 608,651 & 2.71 & Dominated \\
\hline CFZ-LEN-DEX & 492,872 & 3.45 & Dominated & 459,868 & 2.74 & Dominated & 459,868 & 2.74 & Dominated \\
\hline PAN-BOR-DEX & & & & 190,876 & 3.23 & 14,598 & & \\
\hline DAR-BOR-DEX & 447,182 & 5.29 & 50,704 & 423,119 & 4.38 & 248,762 & 423,119 & 4.38 & 60,359 \\
\hline DAR-LEN-DEX & 845,527 & 5.44 & $2,707,547$ & 789,202 & 4.38 & $\begin{array}{c}\text { Equal outcomes, } \\
\text { higher cost vs. } \\
\text { DAR-BOR-DEX }\end{array}$ & 789,202 & 4.38 & $\begin{array}{c}\text { Equal outcomes, } \\
\text { higher cost vs. } \\
\text { DAR-BOR-DEX }\end{array}$ \\
\hline
\end{tabular}

BOR= bortezomib; $C F Z=$ carfilzomib; DAR=daratumumab; DEX = dexamethasone; ELO=elotuzumab; ICER=incremental cost-effectiveness ratio; IX=ixazomib; LEN =lenalidomide; $O S=$ overall survival; PAN = panobinostat; $P F S=$ progression-free survival; $Q A L Y=$ quality-adjusted life-year.

\section{Sensitivity Analyses}

One-way sensitivity analyses used 95\% CIs from clinical evidence when available and plausible values from the published literature when absent. We also conducted a probabilistic sensitivity analysis by jointly varying all model parameters over 4,000 simulations, then calculating 95\% credible range estimates for each model outcome. The following distributions were applied to model variables: hazard ratios (log-normal distribution), utility values (beta distribution), proportions of AEs (beta distribution), costs of drugs (normal distribution), costs of AEs (normal distribution), monthly cost of progression (normal distribution), and administration costs (normal distribution).

We ran 4 scenario analyses: (1) using an unadjusted estimate of the relationship of median PFS to median OS based on a weighted average from the trials in our analysis that report both outcomes (3.27-month increase in OS for each additional month of median PFS); (2) using BOR+DEX as the baseline comparator; (3) adjusting the second- and third-line baseline curves to reflect more recent LEN+DEX regimen curves using the relationship between the ASPIRE trial LEN+DEX data and the MM-009/MM-010 pooled LEN+DEX data,7; and (4) using different second-line utility estimates for triplet $(0.83,0.85$, and 0.66 for PFS on treatment, PFS off treatment, and progression, respectively) versus doublet regimens $(0.81,0.83,0.4$, respectively) derived from the ASPIRE trial data (triplet regimens include 3 drugs [e.g., CFZ+LEN+DEX]; doublet regimens include 2 drugs [e.g., LEN+DEX]., ${ }^{7,18}$

\section{Results}

\section{Network Meta-analysis}

Results of the NMA are included in Table 1. Trial populations were similar with respect to age, ECOG performance status, ISS stage, receipt of previouis stem cell transplant, and number and distribution of previous regimens. (Appendix A). Definitions of disease risk varied, but the percentage of patients with highrisk disease ranged from 13\%-32\% across studies reporting this element. All of the regimens had favorable hazard ratios compared with LEN+DEX; ELO+LEN+DEX and IX+LEN+DEX had the greatest uncertainty. Results for PAN+BOR+DEX in the third-line setting should be interpreted with great caution because of censoring issues and high rates of toxicity-related discontinuation in the overall and third-line subgroup populations of the PANORAMA-1 study. ${ }^{14} \mathrm{PAN}+\mathrm{BOR}+\mathrm{DEX}$ is also only 1 of 2 regimens without direct comparative evidence versus LEN+DEX; therefore, greater reliance on the study network and its assumptions regarding minimal heterogeneity across study populations and constant hazards over time was required. While censoring was factored into our analytic approach, the relative treatment effect of PAN+BOR+DEX versus LEN+DEX had much greater uncertainty than the other comparisons.

\section{Cost-effectiveness}

The results for the aggregate discounted clinical and economic outcomes by line and regimen are provided in Tables 2 and 3 . In the second line, total QALYs ranged from a low of 2.59 for LEN+DEX to a high of 5.44 for DAR+LEN+DEX. Total life years ranged from 3.53 for LEN+DEX to 7.38 for DAR+LEN+DEX. Total costs ranged from $\$ 189,357$ for BOR+DEX to $\$ 845,527$ for DAR+LEN+DEX. In the third line, results followed a similar pattern, with total QALYs ranging from a low of 2.04 for LEN+DEX to a high of 4.38 for DAR+LEN+DEX. Total life years ranged from 3.25 for LEN+DEX to 6.97 for DAR+LEN+DEX. Total costs ranged from $\$ 175,315$ for BOR+DEX to $\$ 789,202$ for DAR+LEN+DEX.

Table 2 shows the comparative results using a league table approach, which listed interventions from lowest to highest QALYs and then calculated an incremental cost-effectiveness ratio (ICER) for each intervention compared with the next best option. Interventions that were dominated were removed from the calculations, and a new ICER was computed versus the next best comparator. Table 2 shows that in the second line, BOR+DEX dominates LEN+DEX; DAR+BOR+DEX has an ICER of $\$ 50,704$ versus BOR+DEX; and DAR+LEN+DEX has 
Cost-effectiveness of Drugs to Treat Relapsed/Refractory Multiple Myeloma in the United States

\begin{tabular}{|c|c|c|c|c|c|c|c|c|c|c|c|}
\hline otal costs, \$ & 309,997 & 189,357 & \multicolumn{2}{|c|}{492,872} & \multicolumn{2}{|c|}{665,728} & \multicolumn{2}{|c|}{622,378} & & 845,527 & 447,182 \\
\hline Supportive care & 528 & 1,608 & \multicolumn{2}{|c|}{1,882} & \multicolumn{2}{|c|}{2,607} & \multicolumn{2}{|r|}{2,491} & & 4,947 & 2,515 \\
\hline Administration & - & 8,226 & \multicolumn{2}{|c|}{8,377} & \multicolumn{2}{|c|}{14,698} & \multicolumn{2}{|r|}{-} & & 23,981 & 22,960 \\
\hline Progression & 40,221 & 41,167 & \multicolumn{2}{|c|}{45,358} & \multicolumn{2}{|c|}{45,143} & \multicolumn{2}{|r|}{44,330} & & 50,723 & 51,003 \\
\hline PFS & 1.41 & 1.50 & 1.9 & & & 1.89 & & 1.81 & & 3.13 & 3.05 \\
\hline Progression & 1.17 & 1.24 & 1.5 & & & 1.52 & & 1.46 & & 2.31 & 2.24 \\
\hline otal life-years & 3.53 & 3.73 & 4.7 & & & 4.66 & & 4.46 & & 7.38 & 7.11 \\
\hline PFS & 1.73 & 1.83 & 2.3 & & & 2.31 & & 2.21 & & 3.82 & 3.67 \\
\hline Progression & 1.80 & 1.91 & 2.3 & & & 2.34 & & 2.25 & & 3.55 & 3.44 \\
\hline CER vs. LEN-DEX & - & $-792,583$ & 211,45 & & 430, & 009 & & 454,684 & & 187,728 & 50,704 \\
\hline Supportive care & 473 & 1,441 & 1,779 & & 2,364 & & 55 & 4 & & 4,579 & 2,403 \\
\hline Administration & & 7,365 & 8,113 & & 3,394 & - & & 3,05 & & 22,394 & 21,412 \\
\hline Progression & 39,261 & 40,175 & 44,318 & & 4,105 & 43, & & 46,74 & & 51,708 & 52,014 \\
\hline Adverse event & 4,351 & 4,583 & 4,457 & & 7,156 & & 66 & 9,12 & & 3,469 & 2,607 \\
\hline otal QALYs & 2.04 & 2.16 & 2.74 & & 2.71 & & 60 & 3.2 & & 4.38 & 4.38 \\
\hline PFS & 1.00 & 1.07 & 1.37 & & 1.36 & & 30 & 1.6 & & 2.28 & 2.35 \\
\hline Progression & 1.03 & 1.09 & 1.37 & & 1.36 & & 30 & 1.5 & & 2.10 & 2.03 \\
\hline otal life-years (OS) & 3.25 & 3.44 & 4.37 & & 4.32 & & .14 & 4.5 & & 6.97 & 6.71 \\
\hline PFS & 1.55 & 1.64 & 2.12 & & 2.09 & & 00 & 2. & & 3.52 & 3.38 \\
\hline Progression & 1.70 & 1.79 & 2.25 & & 2.23 & & .14 & 2.5 & & 3.44 & 3.33 \\
\hline CER vs. LEN-DEX & - & $-853,800$ & 252,293 & & 4,168 & 508, & & Domin & & 216,360 & 60,359 \\
\hline
\end{tabular}

$B O R=$ bortezomib; $C F Z=$ carfilzomib; DAR=daratumumab; $D E X=$ dexamethasone; $E L O=$ elotuzumab; ICER=incremental cost-effectiveness ratio; $I X=i x a z o m i b ;$ $L E N=$ lenalidomide; $O S=$ overall survival; $P A N=$ panobinostat; $P F S=$ progression-free survival; $Q A L Y=$ quality-adjusted life-year.

an ICER of $\$ 2,707,547$ versus DAR+BOR+DEX. In the third line, BOR+DEX dominates LEN+DEX; PAN+BOR+DEX has an ICER of $\$ 14,124$ versus BOR+DEX; DAR+BOR+DEX has an ICER of $\$ 248,762$; and DAR+BOR+DEX is cost minimizing versus DAR+LEN+DEX. If we remove PAN+BOR+DEX because of the challenges mentioned in the Network Meta-analysis section (including high levels of treatment discontinuation in the PANORAMA-1 trial and lack of direct comparative evidence to LEN+DEX, among others), DAR+BOR+DEX has an ICER of $\$ 60,359$ versus $B O R+D E X$.

The results of the pairwise analyses can be found in Table 3. ICERS for new second-line regimens versus LEN+DEX were estimated to be $\$ 51,000$ per QALY for DAR+BOR+DEX, followed by DAR+LEN+DEX $(\$ 188,000)$ and CFZ+LEN+DEX $(\$ 211,000)$, with greater than $\$ 400,000$ per QALY for ELO+LEN+DEX and IX+LEN+DEX. In the third line, ICERs for new regimens versus LEN+DEX were estimated to range from dominant for PAN+BOR+DEX to $\$ 60,000$ per QALY for DAR+BOR+DEX, followed by DAR+LEN+DEX $(\$ 216,000)$, CFZ+LEN+DEX $(\$ 253,000)$, and approximately $\$ 500,000$ per QALY for ELO+LEN+DEX and IX+LEN+DEX. As noted previously, results for PAN+BOR+DEX should be interpreted with caution because of population censoring and reliance on indirect treatment comparisons in the NMA. In both settings, incremental costs were driven primarily by increased drug costs rather than progression, supportive care, or AE costs.

\section{Sensitivity Analyses}

In each one-way analysis (not shown), results were by far most sensitive to the PFS hazard ratios for each intervention versus LEN+DEX, followed by the estimated link between PFS and OS (2.45 months of OS for each month of PFS, per Felix et al., $\left.2013^{24}\right)$, drug costs, dosage intensity, and health state utilities. ${ }^{6}$

Probabilistic sensitivity analysis showed variability in model outcomes (Figure 1). In the second-line setting among the new agents, DAR+BOR+DEX had an $87 \%$ probability of being costeffective at \$150,000 per QALY, while all other agents had a $0 \%$ probability of being cost-effective at that threshold. In the third-line setting, PAN+BOR+DEX had an $87 \%$ probability of being cost-effective at $\$ 150,000$ per QALY; however, this probability declined with increasing willingness to pay for the better survival outcomes of DAR+BOR+DEX. No other new 
Second Line

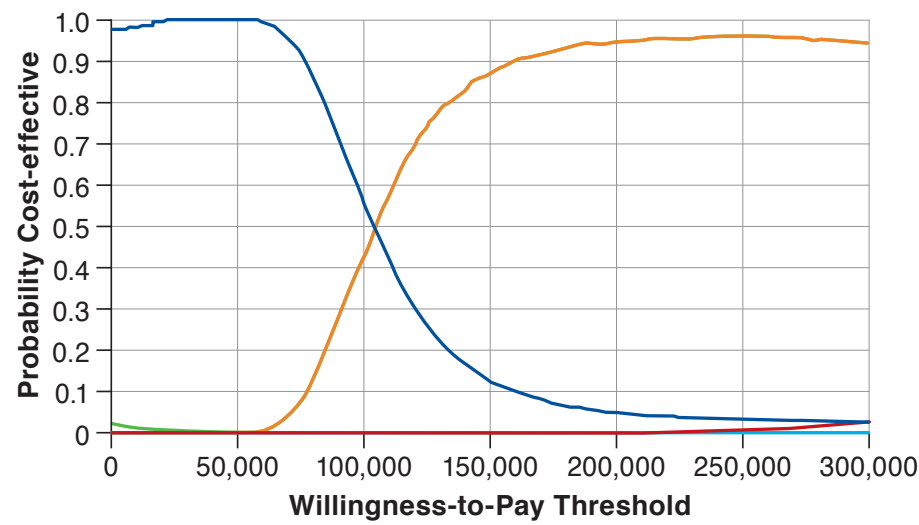

Third Line

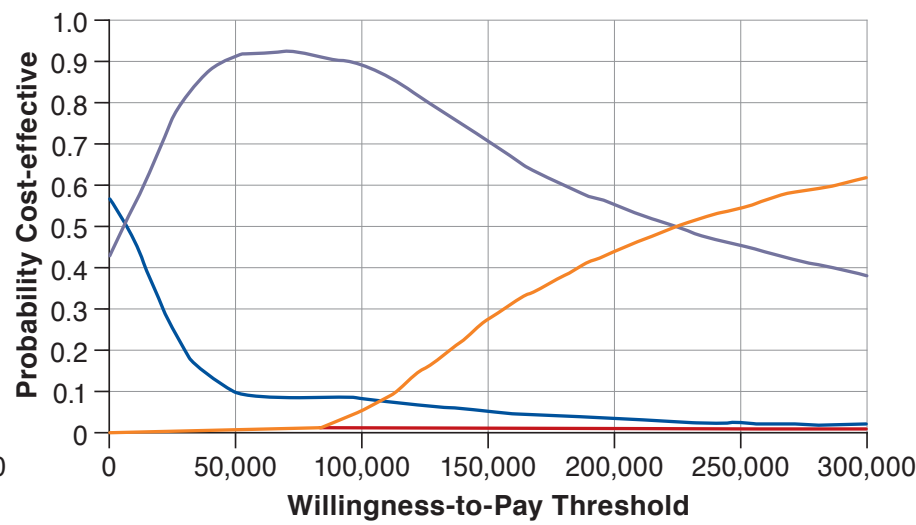

- LEN-DEX —BOR-DEX —CFZ-LEN-DEX —ELO-LEN-DEX

-IX-LEN-DEX —DAR-LEN-DEX

DAR-BOR-DEX

PAN-BOR DEX

BOR=bortezomib; $C F Z=$ carfilzomib; $D A R=$ daratumumab; $D E X=$ dexamethasone; ELO=elotuzumab; $I X=$ ixazomib; LEN =lenalidomide; PAN=panobinostat.

agents were cost-effective at a cost-effectiveness acceptability threshold of \$150,000 per QALY.

We performed several scenario analyses (data not shown). The resulting ICERs varied but no regimens were found to have ICERs below $\$ 150,000$ per QALY that were not already estimated to be so in the primary analysis.

Finally, we performed a threshold analysis to estimate the unit price coincident with commonly used cost-effectiveness thresholds, by holding all other parameters constant (including BOR, LEN, and DEX costs) and identifying the threshold price for each novel agent that made the overall regimen cost-effective at different willingness-to-pay thresholds. We also implemented this analysis with the probabilistic sensitivity analysis to calculate $95 \%$ credible range estimates for each threshold price. Table 4 demonstrates that most agents, in combination regimens, would require substantial discounts to meet the highest cost-effectiveness threshold of $\$ 150,000$ per QALY, and some would not even be cost-effective at a price of $\$ 0$, given the existing cost of the other drugs in the regimen.

\section{Discussion}

We evaluated 8 drugs in 8 regimens for 2 relapsed and/or refractory MM populations, second and third lines. All the regimens evaluated were estimated to increase time in the progression-free health state and overall survival versus the standard regimens of LEN+DEX and BOR+DEX, but at substantial additional cost compared with these doublet regimens. As such, the value for most of these regimens, according to commonly used thresholds for cost-effectiveness, would be considered questionable. A few regimens were estimated to be in the cost-effective range, including DAR-BOR-DEX (second line) and PAN-BOR-DEX (third line), although the analysis for PAN+BOR+DEX was reserved to the third line and had data challenges that limited our willingness to make strong conclusions about its value. If we remove PAN+BOR+DEX from the third-line analysis, DAR-BOR-DEX becomes the most costeffective. The LEN+DEX-based regimens with the best ICERs were DAR+LEN+DEX and CFZ+LEN+DEX, although neither regimen fell below the $\$ 150,000$ per QALY threshold due, in part, to the high cost of LEN and the longer duration of therapy because of increased PFS compared with doublet regimens.

The analyses reported here reveal that important advances in the treatment of relapsed and/or refractory MM have been made over the past decade, which have expanded treatment options and improved patient outcomes. However, only a few regimens have done so in a cost-effective manner.

Our findings highlight a few key implications for stakeholders facing treatment, recommendation, or coverage and reimbursement decisions related to relapsed and/or refractory MM. First, there are cost-effective options for patients in this setting. DAR, the agent with the highest estimated life expectancy and QALY outcomes, demonstrated good value when used in combination with BOR-DEX in the second- and third-line settings. Depending on the clinical situation and patient and provider preferences, this regimen may be preferred over other regimens. Second, new agents combined with BOR uniformly had better ICERs relative to the same new agents in combination with LEN. This is primarily a function of the drug cost for BOR and LEN, respectively. The cost per month for BOR is about half as much as that for LEN. Couple this with the treat-to-progression dosing schedules, and the BOR-based regimens were uniformly more cost-effective compared with the LEN-based regimens. 
Cost-effectiveness of Drugs to Treat Relapsed/Refractory Multiple Myeloma in the United States

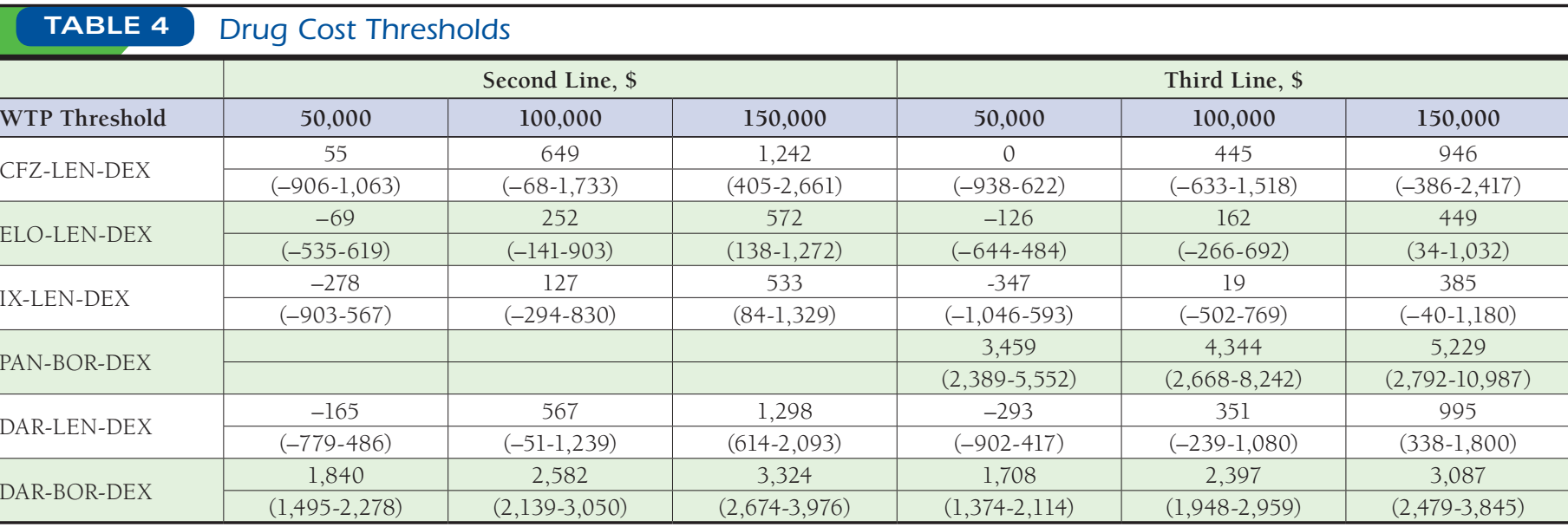

Note: Results reflect threshold prices for the first listed drug in each triplet regimen only (all other parameter values held constant).

$B O R=$ bortezomib; $C F Z=$ carfilzomib; DAR=daratumumab; DEX=dexamethasone; ELO=elotuzumab; IX=ixazomib; LEN =lenalidomide; PAN = panobinostat; WTP = willingness to pay.

Importantly, incremental drug costs included additional costs of the new drug as well as extended use of LEN+DEX or BOR-DEX because of improved PFS for the entire regimen. For example, the total treatment cost of LEN in the preprogression state when given as part of the CFZ+LEN+DEX regimen is $\$ 260,392$ versus $\$ 239,745$ when given as part of the LEN+DEX regimen because of the longer time in the progression-free state and therefore longer time on treatment. With treat-to-progression strategies, the additional clinical benefits of extending time in the progression-free health state come with consistent extra costs, whereas regimens that generally include 1 or more agents with a fixed dosing schedule (i.e., CFZ+LEN+DEX and PAN+BOR+DEX) do not incur the same amount of additional cost. In this context, the cost and value in real-world settings will be different if the clinical community deviates from the fixed dosing strategies suggested in the prescribing information.

The drug cost threshold analysis highlights a current challenge to providing cost-effective cancer treatment over and above that related to the cost of individual drugs, that is, the issue of adding new agents to existing regimens and creating expensive combination regimens of 2 or more drugs. This challenge has been discussed previously and remains a challenge globally. 29,30 Specifically, manufacturers of new drugs that are used in combination with other, often expensive, drugs have no influence over the cost of the other drugs; therefore, manufacturers have a limited ability to establish prices in line with a given health system's willingness to pay for health gains. As our threshold analysis revealed, for many of the drugs evaluated, the discounts that would be required to achieve commonly used cost-effectiveness thresholds were unrealistic and at times entered into the negative space. However, these are real extra costs to the health system and must be factored into economic analyses.

There are limited options available to address this issue. Indication-based pricing has gained some traction as a potential option to align the use of drugs with the specific value delivered to patients and patient populations, but the challenges with creating this system for an individual drug therapy would only be exacerbated for combination regimens. CMS has indicated a willingness to explore innovative contracts, especially in oncology, but for indication-based pricing to work with combination regimens, the payer would need to establish a comprehensive indication-based pricing process that applied to all manufacturers, and we are a long way from such a system at present. ${ }^{31}$ Some companies have indicated that they are willing to entertain decreased costs for drugs used in combination if they make all the drugs. ${ }^{32}$ Another possibility is for manufacturers to work together to provide a group discount. This option has begun to gain traction in Europe, where manufacturers can work with large public payers, although implementation in the United States would be challenged by its fragmented health system. ${ }^{33}$

We found limited data on the cost-effectiveness of drugs to treat MM in the United States. A recently published costeffectiveness analysis by Jakubowiak et al. (2016) examined CFZ+LEN+DEX versus LEN+DEX in relapsed MM from a U.S. perspective. ${ }^{34}$ Although the total incremental cost was similar to that in our model ( $\$ 179,400$ vs. $\$ 183,000$, respectively), the estimate of QALYs gained with CFZ+LEN+DEX was notably different (1.67 vs. 0.86).

This difference appears to be a result of 2 key differences between models. First, the independently modeled PFS and OS curves in the Jakubowiak et al. analysis yielded much more favorable estimates of treatment effect for CFZ+LEN+DEX than those reported in the ASPIRE trial versus LEN+DEX (PFS odds ratio $=0.51$ [model] vs. 0.69 [published hazard ratio]; OS hazard ratio $=0.70$ [model] vs. 0.79 [published hazard ratio]). These differences appear to explain a modeled increase in PFS that was over 5 months longer than the median PFS observed 
in the ASPIRE trial (unpublished data. Amgen. Data provided in response to ICER data request. QOL/Utility Data from ASPIRE Cost Effectiveness Model. February 22, 2016) Second, Jakubowiak et al. used a log-logistic parametric function for the PFS curve, which is prone to long tails in the distribution, whereas we used a Weibull function. Finally, we note that 1 of the findings of the Jakubowiak et al. analysis appears to be counterintuitive, in that CFZ+LEN+DEX patients spend approximately 4 years in the postprogression state in the model versus approximately 3 years for LEN+DEX; however, the postprogression treatment costs for LEN+DEX are reported to be higher.

\section{Limitations}

Our analysis had several limitations that warrant mention. We had limited data for all agents on OS. Therefore, we used a method supported by other methodologists, and used in previous analyses, to estimate OS using PFS. ${ }^{25,35}$ Although directly observed data on OS would be preferred, we felt this method allowed for an unbiased and uniformly applicable approach to estimated OS using PFS, the primary endpoint from all the pivotal trials. This approach would be expected to limit potential bias across drugs given that the agents and regimens evaluated were all approved at different times, so the standard of care, especially after progression, would be expected to vary and potentially affect OS. The observed relationship in any individual study may have been different than that applied uniformly in our model. We therefore tested the estimate in 1-way sensitivity and scenario analyses and found that, while the assumed relationship of PFS to OS was a sensitive parameter, its effect was much less than that of varying PFS hazard ratios and did not substantially affect the primary findings.

We did not have sufficient data for each regimen in the second and third lines. Therefore, we used line-specific data from LEN+DEX, the baseline comparator, and applied the same treatment effect for the new regimens to the separate baseline population estimates, under the assumption that the treatment effect (i.e., the hazard ratio) was consistent across the second and third lines. This assumption had validity in that we found no consistent evidence of a differential treatment effect by line of therapy, and the trials were powered to detect differences in the overall effect in the full intent-to-treat population.

Finally, we note that PFS results in the tables will not match those seen in clinical trials because of our anchoring of hazard ratios to the baseline survival curves for LEN+DEX rather than use of observed survival curves in each trial. However, because of the fixed-effects nature of the NMA, relative effects from each trial are essentially preserved. Our drug cost estimates also had good face validity when compared against an analysis performed by Potluri et al. (2015) using the MarketScan claims database (total LEN+DEX cost in the model: $\$ 280,000$ vs. Potluri et al.: approximately $\$ 310,000){ }^{36}$

\section{Conclusions}

The introduction of newer drugs and regimens to treat second- and third-line relapsed and/or refractory MM appears to provide clinical benefits by lengthening PFS and OS and improving quality of life. However, only the addition of DAR or PAN may be considered cost-effective options according to commonly cited thresholds, and PAN+BOR+DEX results require cautious interpretation. Achieving levels of value more closely aligned with patient benefit would require substantial discounts for the remaining agents evaluated.

\section{Authors}

JOSH J. CARLSON, MPH, PhD; GREGORY F. GUZAUSKAS, MSPH, PhD; and ELIZABETH D. BROUWER, MPH, Pharmaceutical Outcomes Research and Policy Program, University of Washington, Seattle. RICHARD H. CHAPMAN, PhD, MS; PATRICIA G. SYNNOTT, MS, MALD; SHANSHAN LIU, MS; ELIZABETH T. RUSSO, MD; STEVEN D. PEARSON, MD, MSc; and DANIEL A. OLLENDORF, PhD, Institute for Clinical and Economic Review, Boston, Massachusetts.

AUTHOR CORRESPONDENCE: Josh J. Carlson, MPH, PhD, Associate Professor, Pharmaceutical Outcomes Research and Policy Program, University of Washington, 1959 N.E. Pacific St., H-375I Box 357630, Seattle, WA 98195-7630. Tel.: 206.543.9649;

E-mail: Carlsojj@u.washington.edu.

\section{DISCLOSURES}

Funding for this work was provided in part by the Institute for Clinical and Economic Review, which collaborated on the design, conduct, and reporting of this evaluation. During the conduct of this study, Ollendorf, Synnott, Chapman, and Pearson report grants from Blue Shield of California Foundation, California Health Care Foundation, and Laura and John Arnold Foundation and also report other grants from Aetna, AHIP, Anthem, Blue Shield of California, CVS Caremark, Express Scripts, Harvard Pilgrim Health Care, OmedaRx, United Healthcare, Kaiser Permanente, Premera, AstraZeneca, Genentech, GlaxoSmithKline, Johnson \& Johnson, Merck, National Pharmaceutical Council, Takeda, Pfizer, Novartis, Lilly, Spark Therapeutics, Sanofi, Prime Therapeutics, and Health Care Service Corporation outside the submitted work.

Carlson reports grants from the Institute for Clinical and Economic Review during the conduct of the study and personal fees from Seattle Genetics, Genentech, and Pfizer outside the submitted work. Russo, Guzauskas, Liu, and Brouwer have nothing to disclose.

Study concept and design were contributed by Carlson, Guzauskas, and Ollendorf. Guzauskas, Chapman, Synnott, and Liu collected the data, and Carlson, Guzauskas, Chapman, and Ollendorf contributed to data analysis, along with Synnott and Liu. The manuscript was written by Carlson, Guzauskas, and Brouwer, along with Chapman, Synnott, and Ollendorf, and revised by Carlson, Brouwer, and Guzauskas, along with Chapman, Synnott, and Ollendorf.

\section{REFERENCES}

1. National Cancer Institute. Surveillance, Epidemiology, and End Results (SEER) Program. Cancer stat facts: myeloma. 2016. Available at: https://seer. cancer.gov/statfacts/html/mulmy.html. Accessed November 2, 2017.

2. National Comprehensive Cancer Network. NCCN Clinical Practice Guidelines in Oncology: Multiple Myeloma. Version 3.2016. Available at: https://www.nccn.org/professionals/physician_gls/default.aspx\#site. Accessed November 2, 2017. 
3. Roy A, Kish JK, Bloudek L, et al. Estimating the costs of therapy in patients with relapsed and/or refractory multiple myeloma: a model framework. Am Health Drug Benefits. 2015;8(4):204-15.

4. Neumann PJ, Cohen JT, Weinstein MC. Updating cost-effectivenessthe curious resilience of the \$50,000-per-QALY threshold. N Engl J Med. 2014;371(9):796-97.

5. Sutton A, Ades A, Cooper N, Abrams K. Use of indirect and mixed treatment comparisons for technology assessment. Pharmacoeconomics. 2008;26(9):753-67.

6. Stewart AK, Rajkumar SV, Dimopoulos MA, et al. Carfilzomib, lenalidomide, and dexamethasone for relapsed multiple myeloma. N Engl J Med. 2015;372(2):142-52.

7. Weber DM, Chen C, Niesvizky R, et al. Lenalidomide plus dexamethasone for relapsed multiple myeloma in North America. N Engl J Med. 2007;357(21):2133-42

8. Dimopoulos M, Spencer A, Attal M, et al. Lenalidomide plus dexamethasone for relapsed or refractory multiple myeloma. N Engl J Med. 2007;357(21):2123-32

9. Richardson PG, Sonneveld P, Schuster MW, et al. Bortezomib or high-dose dexamethasone for relapsed multiple myeloma. N Engl J Med. 2005:352(24):2487-98

10. Dimopoulos MA, Orlowski RZ, Facon T, et al. Retrospective matchedpairs analysis of bortezomib plus dexamethasone versus bortezomib monotherapy in relapsed multiple myeloma. Haematologica. 2015;100(1):100-06. 11. Palumbo A, Chanan-Khan A, Weisel K, et al. Daratumumab, bortezomib, and dexamethasone for multiple myeloma. N Engl J Med. 2016;375(8):754-66. 12. Dimopoulos MA, Oriol A, Nahi H, et al. Daratumumab, lenalidomide, and dexamethasone for multiple myeloma. N Engl J Med. 2016;375(14):1319-31.

13. Lonial S, Dimopoulos M, Palumbo A, et al. Elotuzumab therapy for relapsed or refractory multiple myeloma. N Engl J Med. 2015;373(7):621-31.

14. San-Miguel JF, Hungria VT, Yoon SS, et al. Panobinostat plus bortezomib and dexamethasone versus placebo plus bortezomib and dexamethasone in patients with relapsed or relapsed and refractory multiple myeloma: a multicentre, randomised, double-blind phase 3 trial. Lancet Oncol. 2014;15(11):1195-206.

15. Moreau P, Masszi T, Grzasko N, et al. Oral Ixazomib, lenalidomide, and dexamethasone for multiple myeloma. N Engl J Med. 2016;374(17):1621-34.

16. Ouwens MJ, Philips Z, Jansen JP. Network meta-analysis of parametric survival curves. Res Synth Methods. 2010;1(3-4):258-71.

17. Hoyle MW, Henley W. Improved curve fits to summary survival data: application to economic evaluation of health technologies. BMC Med Res Methodol. 2011;11:139.

18. Stadtmauer E, Weber D, Dimopoulos M, et al. Lenalidomide in combination with dexamethasone is more effective than dexamethasone at first relapse in relapsed multiple myeloma. Blood. 2006;108(11):3552.

19. San Miguel J, Weisel K, Moreau P, et al. Pomalidomide plus low-dose dexamethasone versus high-dose dexamethasone alone for patients with relapsed and refractory multiple myeloma (MM-003): a randomised, openlabel, phase 3 trial. Lancet Oncol. 2013;14(11):1055-66.

20. San Miguel JF, Weisel K, Song KW, et al. Patient outcomes by prior therapies and depth of response: analysis of MM-003, a phase 3 study comparing pomalidomide plus low-dose dexamethasone (POM plus LoDEX) vs high-dose dexamethasone (HiDEX) in relapsed/refractory multiple myeloma (RRMM). Blood. 2013;122(21):686 [Abstract]. Available at: http://www.bloodjournal.org/ content/122/21/686?sso-checked=true. Accessed November 2, 2017.

21. U.S. Food and Drug Administration. Empliciti medical/statistical review (761035Origls000). 2015. Available at: https://www.accessdata.fda.gov/ drugsatfda_docs/nda/2015/761035Origls000MedR.pdf. Accessed November 2, 2017.

22. Avet-Loiseau H, Fonseca R, Siegel D, et al. Efficacy and safety of carfilzomib, lenalidomide, and dexamethasone vs lenalidomide and dexamethasone in patients with relapsed multiple myeloma based on cytogenetic risk status: subgroup analysis from the phase 3 study aspire (NCT01080391). Blood. 2015;126(23):731 [Abstract]. Available at: http://www.bloodjournal.org/ content/126/23/731?sso-checked=true. Accessed November 2, 2017.
23. Moreau P, Masszi T, Grzasko N, et al. Ixazomib, an investigational oral proteasome inhibitor (PI), in combination with lenalidomide and dexamethasone (IRd), significantly extends progression-free survival (PFS) for patients (Pts) with relapsed and/or refractory multiple myeloma (RRMM): the phase 3 Tourmaline-MMl study (NCT01564537). Blood. 2015;126(23):727 [Abstract]. Available at: http://www.bloodjournal.org/ content/126/23/727?sso-checked=true. Accessed November 2, 2017.

24. Felix J, Aragao F, Almeida JM, et al. Time-dependent endpoints as predictors of overall survival in multiple myeloma. BMC Cancer. 2013;13:122.

25. National Institute for Health and Care Excellence (NICE). Pomalidomide for relapsed and refractory multiple myeloma previously treated with lenalidomide and bortezomib. Technology appraisal guidance [TA338] March 25, 2015. Available at: https://www.nice.org.uk/guidance/ta338. Accessed November 2, 2017.

26. Center for Medicare \& Medicaid Services. Acute inpatient prospective payment system. 2015. Available at: http://www.cms.hhs.gov/ AcuteInpatientPPS. Accessed November 2, 2017.

27. Center for Medicare \& Medicaid Services. Physician fee schedule. 2016. Available at: https://www.cms.gov/Medicare/Medicare-Fee-for-ServicePayment/PhysicianFeeSched/PFS-Relative-Value-Files-Items/RVU16A.htm 1?DLPage $=1 \& D L E n t r i e s=10 \& D L S o r t=0 \& D L S o r t D i r=$ descending. Accessed November 20, 2017.

28. Farr AM, Stott-Miller M, Varker H, Spencer D, Shah M, Chen C. Treatment sequencing patterns associated with elderly patients with relapsed/refractory multiple myeloma (MM) in the U.S. community setting. Blood. 2015;126(23):5392 [Abstract]. Available at: http://www.bloodjournal. org/content/126/23/5392. Accessed November 2, 2017.

29. Davis S. Assessing technologies that are not cost-effective at a zero price. Decision Support Unit, School of Health and Related Research, University of Sheffield. July 2014. Available at: https://www.ncbi.nlm.nih.gov/pubmedhealth/PMH0088909/pdf/PubMedHealth_PMH0088909.pdf. Accessed November 2, 2017.

30. National Institute for Health and Care Excellence. Breast cancer (HER2 positive, metastatic)-pertuzumab (with trastuzumab and docetaxel) [ID523]. July 2012. Available at: https://www.nice.org.uk/guidance/indevelopment/gid-tag322. Accessed November 20, 2017.

31. Centers for Medicare \& Medicaid Services. CMS proposes to test new Medicare Part B prescription drug models to improve quality of care and deliver better value for Medicare beneficiaries. March 8, 2016. Available at: https://www.cms.gov/Newsroom/MediaReleaseDatabase/Fact-sheets/2016Fact-sheets-items/2016-03-08.html. Accessed November 20, 2017.

32. LaMattina J. Pfizer offers hope for lower-priced cancer drug combos. Forbes. May 26, 2016. Available at: http://www.forbes.com/sites/johnlamattina/2016/05/26/pfizer-offers-hope-for-lower-priced-cancer-drug-combos/ - 55e2fda0le15. Accessed November 2, 2017.

33. Peter Loftus. Combination drug therapies for cancer show promise at higher potential cost. Wall Street Journal. June 5, 2016. Available at: https:// www.wsj.com/articles/combination-drug-therapies-for-cancer-show-promiseat-higher-potential-cost-1465141936. Accessed November 2, 2017.

34. Jakubowiak AJ, Campioni M, Benedict A, et al. Cost-effectiveness of adding carfilzomib to lenalidomide and dexamethasone in relapsed multiple myeloma from a U.S. perspective. J Med Econ. 2016;19(11):1061-74.

35. Davis S, Tappenden P, Cantrell A. Report by the decision support unit: a review of studies examining the relationship between progression-free survival and overall survival in advanced or metastatic cancer. Decision Support Unit, School of Health and Related Research, University of Sheffield. August 2012. Available at: https://www.ncbi.nlm.nih.gov/pubmedhealth/PMH0092942/pdf/PubMedHealth_PMH0092942.pdf. Accessed November 2, 2017.

36. Potluri R, Farr AM, Hirji I, Davis C, Bhandari H, Oukessou A. Treatment sequencing patterns and costs of care in patients with relapsed/refractory multiple myeloma. Value Health. 2015;18(7):A450 [Abstract PCH118]. Available at: http://www.valueinhealthjournal.com/article/S10983015(15)03210-6/pdf. Accessed November 2, 2017.

37. Acaster S, Gaugris S, Velikova G, Yong K, Lloyd A. Impact of the treatment-free interval on health-related quality of life in patients with multiple myeloma: a UK cross-sectional survey. Supportive Care Cancer. 2013;21(2):599-607. 


\section{APPENDIX A Key Trials Included in the Network Meta-analysis}

\begin{tabular}{|c|c|c|c|c|}
\hline Key Trials & Patient Characteristics & Treatment & Comparator & Harms (Treatment Arm) \\
\hline \multirow{4}{*}{$\begin{array}{l}\text { ASPIRE } \\
\text { Open-label RCT } \\
\text { Phase } 3 \\
\text { Carfilzomib (CFZ) }\end{array}$} & \multirow{4}{*}{$\begin{array}{l}\text { - } \text { Median age: } 64 \\
\text { - ECOG=2: } 9.5 \% \\
\text { - ISS Stage III: } 20 \% \\
\text { - Previous SCT: } 57 \% \\
\text { - High risk: } 12.6 \% \\
\text { - Prior regimens (median): } 2 \\
\text { - Prior BOR: } 65.8 \% \\
\text { - Prior LEN: } 19.8 \%\end{array}$} & $\begin{array}{l}\text { CFZ+LEN+DEX } \\
\quad(n=396)\end{array}$ & $\begin{array}{l}\text { LEN+DEX } \\
(n=396)\end{array}$ & \multirow{4}{*}{$\begin{array}{l}\text { - Discontinued d/t AEs: } 15 \% \\
\text { - SAEs: } 60 \% \\
\text { - Tx-related deaths: } 2 \%\end{array}$} \\
\hline & & - Median f/u: $32.3 \mathrm{~m}$ & - Median f/u: 31.5 m & \\
\hline & & \multicolumn{2}{|c|}{$\begin{array}{l}\text { - OS HR: } 0.79 \text { (95\% CI: } 0.63-0.99 ; P=0.04) \\
\text { - PFS HR: } 0.69 \text { (95\% CI: } 0.57-0.83)\end{array}$} & \\
\hline & & $\begin{array}{l}\text { - Median PFS: } 26.3 \mathrm{~m} \\
\text { - ORR: } 87.1 \%\end{array}$ & $\begin{array}{l}\text { - Median PFS: } 17.6 \mathrm{~m} \\
\text { - ORR: } 66.7 \%, P<0.001\end{array}$ & \\
\hline \multirow{5}{*}{$\begin{array}{l}\text { CASTOR } \\
\text { Open-label RCT } \\
\text { Phase } 3 \\
\text { Daratumumab (DAR) }\end{array}$} & \multirow{5}{*}{$\begin{array}{l}\text { - Median age: } 64 \\
\text { - ECOG=2: NR } \\
\text { - ISS Stage III: } 22 \% \\
\text { - Previous SCT: } 61 \% \\
\text { - del(17p): } 10 \% \\
\text { - Prior regimens (median): } 2 \\
\text { - Prior BOR: } 66 \% \\
\text { - Prior LEN: } 76 \%\end{array}$} & $\begin{array}{l}\text { DAR+BOR+DEX } \\
\quad(n=251)\end{array}$ & $\begin{array}{c}\text { BOR+DEX } \\
(n=247)\end{array}$ & \multirow{5}{*}{$\begin{array}{l}\text { - Discontinued d/t AEs: } 7 \% \\
\text { - SAEs: } 76 \% \\
\text { - Tx-related deaths: } 5 \%\end{array}$} \\
\hline & & \multicolumn{2}{|l|}{ - Median f/u: $7.4 \mathrm{~m}$} & \\
\hline & & - Deaths: $11.6 \%$ & - Deaths: $14.6 \%$ & \\
\hline & & \multicolumn{2}{|c|}{ - PFS HR: 0.39 (95\% CI: 0.28-0.53; P<0.001) } & \\
\hline & & $\begin{array}{l}\text { - Median PFS: NR } \\
\text { - ORR: } 82.9 \%\end{array}$ & $\begin{array}{l}\text { - Median PFS: } 7.2 \mathrm{~m} \\
\text { - ORR: } 63.2 \%\end{array}$ & \\
\hline \multirow{5}{*}{$\begin{array}{l}\text { POLLUX } \\
\text { Open-label RCT } \\
\text { Phase } 3 \\
\text { Daratumumab (DAR) }\end{array}$} & \multirow{5}{*}{$\begin{array}{l}\text { - Median age: } 65 \\
\text { - ECOG=2: } 5 \% \\
\text { - ISS Stage III: } 20 \% \\
\text { - Previous SCT: } 63 \% \\
\text { - del(17p): } 8 \% \\
\text { - Prior regimens (median): } 1 \\
\text { - Prior BOR+LEN: } 15 \%\end{array}$} & $\begin{array}{l}\text { DAR+LEN+DEX } \\
\quad(n=286)\end{array}$ & $\begin{array}{l}\text { LEN+DEX } \\
(n=283)\end{array}$ & \multirow{5}{*}{$\begin{array}{l}\text { - Discontinued d/t AEs: } 8 \% \\
\text { - SAEs: } 49 \% \\
\text { - Tx-related deaths: } 4 \%\end{array}$} \\
\hline & & \multicolumn{2}{|l|}{ - Median f/u: $13.5 \mathrm{~m}$} & \\
\hline & & - Deaths: $10.5 \%$ & - Deaths: $15.9 \%$ & \\
\hline & & \multicolumn{2}{|c|}{ - PFS HR: 0.37 (95\% CI: 0.27-0.52; P<0.001) } & \\
\hline & & $\begin{array}{l}\text { - Median PFS: NR } \\
\text { - ORR: } 92.9 \%\end{array}$ & $\begin{array}{l}\text { - Median PFS: } 18.4 \mathrm{~m} \\
\text { - ORR: } 76.4 \%\end{array}$ & \\
\hline \multirow{3}{*}{$\begin{array}{l}\text { ELOQUENT-2 } \\
\text { Open-label RCT } \\
\text { Phase } 3 \\
\text { Elotuzumab (ELO) }\end{array}$} & \multirow{3}{*}{$\begin{array}{l}\text { - Median age: } 66 \\
\text { - ECOG=2: } 9 \% \\
\text { - ISS Stage III: } 21 \% \\
\text { - Previous SCT: } 54 \% \\
\text { - del(17p): } 32 \% \\
\text { - Prior regimens (median): } 2 \\
\text { - Prior BOR: } 70 \% \\
\text { - Prior LEN: } 6 \%\end{array}$} & $\begin{array}{l}\text { ELO+LEN+DEX } \\
\quad(n=321)\end{array}$ & $\begin{array}{l}\text { LEN+DEX } \\
(n=325)\end{array}$ & \multirow{3}{*}{$\begin{array}{l}\text { - Discontinued d/t AEs: } 13 \% \\
\text { - SAEs: } 65 \% \\
\text { - Tx-related deaths: } 2 \%\end{array}$} \\
\hline & & \multicolumn{2}{|c|}{$\begin{array}{l}\text { - Median f/u: } 24.5 \mathrm{~m} \\
\text { - OS HR: } 0.71 \text { (95\% CI: 0.54-0.93) } \\
\text { - PFS HR: } 0.70 \text { (95\% CI: 0.57-0.85; P<0.001) }\end{array}$} & \\
\hline & & $\begin{array}{l}\text { - Median PFS: } 19.4 \mathrm{~m} \\
\text { - ORR: } 79 \%\end{array}$ & $\begin{array}{l}\text { - Median PFS: } 14.9 \mathrm{~m} \\
\text { - ORR: } 66 \%, P<0.001\end{array}$ & \\
\hline \multirow{5}{*}{$\begin{array}{l}\text { TOURMALINE-MMI } \\
\text { Double-blind RCT } \\
\text { Phase } 3 \text { (unpublished) } \\
\text { Ixazomib (IX) }\end{array}$} & \multirow{5}{*}{$\begin{array}{l}\text { - Median age: } 66 \\
\text { - ECOG=2: } 6 \% \\
\text { - ISS Stage III: } 13 \% \\
\text { - Previous SCT: } 57 \% \\
\text { - High risk: } 19 \% \\
\text { - Prior regimens (median): } 2 \\
\text { - Prior BOR: } 69 \% \\
\text { - Prior LEN: } 12 \%\end{array}$} & $\begin{array}{l}\text { IX+LEN+DEX } \\
(n=360)\end{array}$ & $\begin{array}{l}\text { Placebo+LEN+DEX } \\
(n=362)\end{array}$ & \multirow{5}{*}{$\begin{array}{l}\text { - Discontinued d/t AEs: } 13 \% \\
\text { - SAEs: } 40 \% \\
\text { - Tx-related deaths: NR }\end{array}$} \\
\hline & & \multicolumn{2}{|l|}{ - Median f/u (PFS): $23 \mathrm{~m}$} & \\
\hline & & - Deaths: $22.5 \%$ & - Deaths: $24.8 \%$ & \\
\hline & & \multicolumn{2}{|c|}{ - PFS HR: 0.74 (95\% CI: 0.59-0.94; $P=0.012)$} & \\
\hline & & $\begin{array}{l}\text { - Median PFS: } 20.6 \mathrm{~m} \\
\text { - ORR: } 78 \%\end{array}$ & $\begin{array}{l}\text { - Median PFS: } 14.7 \mathrm{~m} \\
\text { - ORR: } 72 \%, P<0.001\end{array}$ & \\
\hline \multirow{4}{*}{$\begin{array}{l}\text { PANORAMA-1 } \\
\text { Double-blind RCT } \\
\text { Phase } 3 \\
\text { Panobinostat (PAN) }\end{array}$} & \multirow{4}{*}{$\begin{array}{l}\text { - Median age: } 63 \\
\text { - ECOG=2: } 5 \% \\
\text { - ISS Stage III: } 22 \% \\
\text { - Previous SCT: } 58 \% \\
\text { - } 1 \text { prior regimen: } 51 \% \\
\text { - Prior BOR+DEX: } 38 \% \\
\text { - Prior LEN: } 21 \%\end{array}$} & $\begin{array}{l}\text { PAN+BOR+DEX } \\
\quad(n=387)\end{array}$ & $\begin{array}{l}\text { Placebo+BOR+DEX } \\
(n=381)\end{array}$ & \multirow{4}{*}{$\begin{array}{l}\text { - Discontinued d/t AEs: } 36 \% \\
\text { - SAEs: } 60 \% \\
\text { - Tx-related deaths: } 3 \%\end{array}$} \\
\hline & & - Median f/u: $6.4 \mathrm{~m}$ & - Median f/u: 5.9 m & \\
\hline & & \multicolumn{2}{|c|}{$\begin{array}{l}\text { - OS HR: } 0.87(95 \% \text { CI: } 0.69-1.10 ; P=0.26) \\
\text { - PFS HR: } 0.63 \text { (95\% CI: } 0.52-0.76 ; P<0.0001)\end{array}$} & \\
\hline & & $\begin{array}{l}\text { - Median PFS: } 11.99 \mathrm{~m} \\
\text { - ORR: } 60.7 \%\end{array}$ & $\begin{array}{l}\text { - Median PFS: } 8.08 \mathrm{~m} \\
\text { - ORR: } 54.6 \%, P=0.09\end{array}$ & \\
\hline
\end{tabular}

$A E=$ adverse event $B O R=$ bortezomib; $C I=$ confidence interval; del $(17 p)=$ deletion in 17 region of tumor protein 53 gene; DEX $=$ dexamethasone; $d / t=$ due to;

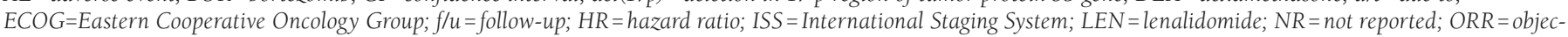

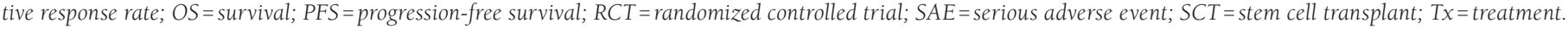


APPENDIX B Grade 3/4 Adverse Event Rates 3,26

\begin{tabular}{|c|c|c|c|c|c|c|c|c|c|}
\hline & $\begin{array}{c}\text { LEN-DEX } \\
\%\end{array}$ & $\begin{array}{c}\text { BOR-DEX } \\
\%\end{array}$ & $\begin{array}{c}\text { CFZ- } \\
\text { LEN-DEX } \\
\%\end{array}$ & $\begin{array}{c}\text { ELO- } \\
\text { LEN-DEX } \\
\%\end{array}$ & $\begin{array}{c}\text { IX- } \\
\text { LEN-DEX } \\
\%\end{array}$ & $\begin{array}{c}\text { PAN- } \\
\text { BOR-DEX } \\
\%\end{array}$ & $\begin{array}{c}\text { DAR- } \\
\text { LEN-DEX } \\
\%\end{array}$ & $\begin{array}{c}\text { DAR- } \\
\text { BOR-DEX } \\
\%\end{array}$ & $\begin{array}{c}\text { Per } \\
\text { AE Cost } \\
\$\end{array}$ \\
\hline & $\mathrm{n}=353$ & $\mathrm{n}=64$ & $\mathrm{n}=392$ & $\mathrm{n}=318$ & $\mathrm{n}=360$ & $\mathrm{n}=381$ & $\mathrm{n}=360$ & $\mathrm{n}=381$ & \\
\hline Anemia & 17.50 & 19.50 & 14.80 & 18.90 & 11.70 & 17.80 & 12.40 & 14.40 & 971 \\
\hline Arrythmias & 3.00 & 2.00 & NR & NR & 5.50 & 3.00 & NR & NR & 6,998 \\
\hline Back pain & 3.00 & 3.00 & NR & 5.00 & 0.80 & 0.80 & 1.40 & NR & 10,728 \\
\hline Cataract & 2.80 & NR & NR & 6.30 & NR & NR & NR & NR & 3,700 \\
\hline Deep vein thrombosis & 3.40 & NR & 4.10 & 5.70 & 3.00 & NR & 1.80 & NR & 31,645 \\
\hline Diarrhea & 3.50 & 8.00 & 3.80 & 5.00 & 6.40 & 25.50 & 5.30 & 3.70 & 9,738 \\
\hline Fatigue & 5.10 & 11.90 & 7.70 & 12.60 & 4.00 & 23.90 & 6.40 & 4.50 & 8,437 \\
\hline Hyperglycemia & 6.30 & NR & 4.60 & 17.00 & 2.20 & NR & NR & NR & 166 \\
\hline Hypertension & 1.50 & NR & 4.30 & NR & 3.00 & NR & NR & 6.60 & 5,478 \\
\hline Hypocalcemia & 3.60 & 2.00 & 2.60 & 11.30 & 4.40 & 5.00 & NR & NR & 1,155 \\
\hline Hypokalemia & 5.20 & 7.00 & 10.50 & 11.30 & 4.40 & 18.00 & NR & NR & 1,707 \\
\hline Lymphopenia & 27.40 & 40.20 & 46.40 & 76.70 & 32.50 & 53.60 & 5.30 & 9.50 & 166 \\
\hline Nausea & 0.40 & 0.50 & 0.20 & 0.90 & 1.70 & 5.50 & 1.40 & NR & 11,934 \\
\hline Neutropenia & 34.70 & 11.40 & 38.80 & 33.60 & 21.90 & 34.50 & 51.90 & 12.80 & 166 \\
\hline Peripheral/sensory neuropathy & 1.50 & 14.60 & 1.70 & 3.80 & 2.50 & 17.60 & NR & 4.50 & 783 \\
\hline Pneumonia & 8.10 & 10.30 & 8.90 & 14.20 & 6.00 & 12.60 & 7.80 & 8.20 & 14,855 \\
\hline Thrombocytopenia & 14.30 & 31.40 & 25.80 & 19.20 & 25.30 & 67.30 & 12.70 & 45.30 & 166 \\
\hline Vomiting & 0.70 & 1.30 & NR & 0.30 & 1.10 & 7.30 & 1.10 & NR & 11,934 \\
\hline
\end{tabular}

